\title{
Is there any effect of insulin resistance on male reproductive system?
}

\author{
Ayhan Verit ${ }^{1}$, Fatma Ferda Verit ${ }^{2}$, Halil Oncel ${ }^{3}$, Halil Ciftci ${ }^{4}$ \\ ${ }^{1}$ Fatih Sultan Mehmet Research \& Training Hospital, Dept. of Urology, Istanbul, Turkey; \\ 2 Suleymaniye Maternity, Research \& Training Hospital, Dept. of Obstetrics \& Gynaecology, \\ Infertility Research \& Treatment Centre, Istanbul, Turkey; \\ ${ }^{3}$ Sanliurfa Research \& Training Hospital, Dept. of Urology, Sanliurfa, Turkey; \\ ${ }^{4}$ Harran University, School of Medicine, Dept. of Urology, Sanliurfa, Turkey.
}

\begin{abstract}
Summary Objectives: To investigate the possible
effect of insulin resistance (IR) on male reproductive system via evaluation of semen analysis, male sex hormones and serum lipid profiles, and testicular volumes.

Methods: After the exclusions, a total of 80 male patients were enrolled in this prospective study. Body Mass Index (BMI), Testicular volume, semen samples, serum hormone/lipid profiles, high sensitive C-Reactive Protein (hsCRP) were obtained from all the subjects. Results: The patients were divided into two groups as study and control according to the presence of IR. There were no statistical differences in terms of age, marriage period, testicular volume, serum levels of hormone and lipid profiles and BMI between the groups. There were no relationship between homeostasis model assessment of insulin resistance (HOMA-IR) and semen volume $(r=-0.10, p=0.37)$, total sperm count $(r=-0.09$, $p=0.39)$, motility $(r=-0.15, p=0.16)$ and morphology $(r=-0.14, p=0.19)$. However, HOMA-IR was closely associated with hsCRP levels $(r=0.94, p<0.0001)$. Conclusions: Despite of the documented strong inverse relationships between Diabetes Mellitus (DM) and male/female fertility, and also between IR and female infertility via ovarian functions as in polycystic ovary syndrome, to our knowledge, there is no report about any influence of IR on male fertility. DM and metabolic syndrome (MetS) have negative influence on fertility. Thus, IR may be accused of causing detrimental effect on male infertility due to hyperinsulinemic state and being one of the components for MetS. Interestingly, due to our preliminary results, we do not found any inverse correlation between IR and male reproductive functions.
\end{abstract}

KEY WORDS: Insulin resistance; Male reproductive system; Male fertility; Sex hormones; sperm.

Submitted 22 September 2013; Accepted 5 October 2013

\section{INTRODUCTION}

The impaired glucose metabolism as in Diabetes mellitus (DM) is widely accepted as a negative effecting factor on human fertility in either gender $(1,2)$. However this detrimental effect of DM on male infertility has long been regarded as a controversial topic due to the fact that the literature knowledge mostly depended on the conventional semen analysis which is not a well predictor of male fertility $(3,4)$. Nevertheless, it was reported that DM can inversely effect male fertility via sperm functions at molecular level especially on its nuclear and mitochondrial DNA and also its repairing systems (3). Moreover, DM is responsible for a kind of histological damage of the epididymis that resulted in a negative impact on sperm transit and also promotes inflammatory process in sexual male accessory glands (5). According the relatively new issue of the endless story of glucose metabolism, insulin resistance (IR), is defined as elevated serum insulin levels due to the end of organ resistance at molecular level with reduced glucose uptake (6). IR and hyperinsulinemia play a major role in the pathogenesis of type 2 diabetes (DM2). IR is claimed of inverse effect on female health such as infertility via ovarian functions as in polycystic ovary syndrome (PCOS), increased risk pregnancy and elevated lifetime risk of developing Type 2 diabetes (7). IR should be taken into account in the definition of metabolic syndrome (MetS) which represents a group of abnormalities, including overweight, dyslipidemia, hypertension, endothelial dysfunction, systemic inflammation and impaired glucose metabolism that reflects our modern's world sedentary lifestyle accompanying with over nutrition and thus became one of the major public health challenges worldwide $(8,9)$.

To our interest, the harm of MetS on male infertility is mostly attributed to the various problems related with overweight such as high scrotal temperature, variations of serum testosterone levels or dyslipidemia other than IR component of MetS (9). Despite of the documented well known aforementioned strong inverse relationships between DM and male/female fertility, and also IR and female fertility, to our knowledge, there is no report about any influence of IR on male fertility. Thus, in this preliminary study we investigate the possible effect of IR on male reproductive system via evaluation of semen analysis, male sex hormones and serum lipid profiles, and testicular volumes. 


\section{MATERIALS AND METHODS}

A total of 80 male patients who attended outpatient infertility clinic of Urology Department of our University Hospital between January 2009 and June 2012 were enrolled in this prospective study. This study was approved by University's Institutional Review Board and all the participants signed the informed consent before the onset of study. A detailed medical history and Body Mass Index (BMI) were obtained from all participants. The patients over 40 years, with known erectile dysfunction, chronic/hereditary disease (including prostatitis, hypertension, dyslipidemia needing medical care), malignancy, and smokers, alcohol drinkers, drug abusers, azoospermics and patients who had varicocele were excluded. Systemic and genital examination was performed for all subjects. Testicular volume was measured by ultrasound and calculated as the formula of 0.71 $\mathrm{x}$ length $\mathrm{x}$ width $\mathrm{x}$ thickness. The patients were divided into two groups as study and control according to the presence of IR or not respectively. The blood samples were undertaken at 9:00 and 11:00 a.m. after an overnight fast (at least 12 hours) and centrifuged within $2 \mathrm{~h}$ after withdrawal and assessed on the same day. IR was determined by the homeostasis model assessment of insulin resistance (HOMA-IR) via the formula: fasting bloo sugar $(\mathrm{mg} / \mathrm{dL}) \times$ fasting insulin $(\mu \mathrm{IU} / \mathrm{mL}) / 405$. A HOMA value above 4 was accepted for indication of IR. Insulin was determined by electrochemiluminescent immunoassay (Siemens Immulite 2000, Los Angeles, USA). Gonadotropins, testosterone and high sensitive C-Reactive Protein (hsCRP) levels were also obtained for all subjects. Two semen samples were evaluated in two weeks intervals. Semen samples collected by masturbation in a clean specimen container after a sexual abstinence for 25 days were allowed to liquefy at $37^{\circ} \mathrm{C}$ and evaluated immediately according to WHO recommendations including ejaculate volume, $\mathrm{pH}$, time to liquefaction, sperm concentration, motility and morphology. Sperm concentration was expressed as 106 per millilitre of semen, whereas motility and morphology were expressed as percentage.

\section{Statistical analysis}

Baseline characteristics of the groups were presented as the mean $\pm S D$. Variables with a skewed distribution were log transformed for all analyses. Demographic, laboratory and semen parameters between the study and control groups were assessed by Student's $t$ test. Correlations between HOMA-IR, semen parameters and hsCRP, were assessed by Pearson correlation analysis. $\mathrm{P}<0.05$ was considered as statistically significant.

\section{Results}

The results of 80 male subjects were evaluated. Of those, 40 were diagnosed with IR due to HOMA-IR and considered as study group and remaining 40 formed the control group. The data collection was stopped when each group reached to 40 . Median age of the patients with or without IR were $31.2 \pm 5.0$ (range 18-40) and $29.6 \pm 5.0$ (range 18-39) respectively. There were no statistical differences in terms of age, marriage period, testicular volume and BMI between the groups (Table 1).

Semen and serologic characteristics (hormone and lipid profiles, glucose/insulin, HsCRP levels and HOMA-IR) of the groups were also summarized in Table 2.

\section{Table 1.}

Age, Body mass index (BMI) and testicular volume of patients who presented to infertility center with/without insulin resistance.

\begin{tabular}{|l|c|c|c|}
\hline & $\begin{array}{c}\text { Subjects with IR } \\
(\mathbf{n}=\mathbf{4 0})\end{array}$ & $\begin{array}{c}\text { Subjects without IR } \\
\mathbf{( n = 4 0 )}\end{array}$ & $\mathbf{p}$ \\
\hline Age (year) & $31.2 \pm 5.0$ & $29.6 \pm 5.0$ & 0.15 \\
$\mathrm{BMI}\left(\mathrm{kg} / \mathrm{m}^{2}\right)$ & $25.6 \pm 3.3$ & $25.0 \pm 3.3$ & 0.44 \\
Testicular volume $\left(\mathrm{cm}^{3}\right)$ & $20.2 \pm 3.2$ & $20.9 \pm 3.1$ & 0.33 \\
\hline BMI: Body mass index; IR: Insulin resistance.
\end{tabular}

Table 2.

Laboratory and semen characteristics of patients who presented to infertility center with/without insulin resistance.

\begin{tabular}{|c|c|c|c|}
\hline & $\begin{array}{l}\text { Subjects with IR } \\
\qquad(\mathrm{n}=40)\end{array}$ & $\begin{array}{l}\text { Subjects without IR } \\
\qquad(n=40)\end{array}$ & $p$ \\
\hline FSH (mIU/mL) & $5.8 \pm 2.7$ & $6.4 \pm 2.7$ & 0.36 \\
\hline $\mathrm{LH}(\mathrm{mlU} / \mathrm{mL})$ & $7.8 \pm 2.7$ & $6.9 \pm 2.7$ & 0.14 \\
\hline Total testosterone(ng/dL) & $403.4 \pm 66.2$ & $427.9 \pm 67.4$ & 0.10 \\
\hline SHBG (nmol/L) & $23.9 \pm 4.4$ & $25.4 \pm 4.4$ & 0.13 \\
\hline $\mathrm{TG}(\mathrm{mg} / \mathrm{dL})$ & $158.2 \pm 21.1$ & $116.7 \pm 20.3$ & $<0.0001$ \\
\hline TC (mg/dL) & $191.2 \pm 28.8$ & $166.3 \pm 28.0$ & $<0.0001$ \\
\hline $\mathrm{HDL}(\mathrm{mg} / \mathrm{dL})$ & $38.2 \pm 4.0$ & $44.2 \pm 4.2$ & $<0.0001$ \\
\hline LDL (mg/dL) & $124.4 \pm 20.0$ & $105.9 \pm 19.8$ & $<0.0001$ \\
\hline Fasting glucose (mg/dL) & $111.3 \pm 9.7$ & $89.4 \pm 9.5$ & $<0.0001$ \\
\hline Fasting insulin $(\mu \mathrm{lU} / \mathrm{mL})$ & $22.5 \pm 4.4$ & $6.5 \pm 4.3$ & $<0.0001$ \\
\hline HOMA-IR & $6.1 \pm 1.2$ & $1.4 \pm 0.9$ & $<0.0001$ \\
\hline hsCRP (mg/L) & $3.1 \pm 1.1$ & $0.9 \pm 0.4$ & $<0.0001$ \\
\hline \multicolumn{4}{|l|}{ Semen parameters } \\
\hline Volume (ml) & $2.6 \pm 1.2$ & $2.8 \pm 1.3$ & 0.50 \\
\hline $\begin{array}{l}\text { Total sperm count } \\
\quad(\text { sperm× } 106 / \mathrm{mL})\end{array}$ & $62.4 \pm 39.1$ & $72.1 \pm 39.5$ & 0.27 \\
\hline Motility (\%) & $56.1 \pm 15.6$ & $60.8 \pm 15.5$ & 0.18 \\
\hline Morphology (\%) & $42.7 \pm 9.4$ & $45.7 \pm 9.4$ & 0.16 \\
\hline
\end{tabular}

SHBG: Sex hormone binding globulin; TG: Triglyceride; TC: Total cholesterol; HDL: High density lipoprotein; LDL: Low density lipoprotein; HOMA-IR: Homeostasis model assessment of insulin resistance; hsCRP: High sensitive; C-reactive protein; IR: Insulin resistance. 
There were no relationship between HOMA-IR and semen volume $(r=-0.10, p=0.37)$, total sperm count $(r=-0.09$, $\mathrm{p}=0.39)$, motility $(\mathrm{r}=-0.15, \mathrm{p}=0.16)$ and morphology $(\mathrm{r}=-0.14, \mathrm{p}=0.19)$. However, HOMA-IR was closely associated with hsCRP levels $(r=0.94, p<0.0001)$.

\section{Discussion}

Although IR with related hyperinsulinemia classically is defined as a decreased sensitivity to the metabolic actions of insulin such as insulin-mediated glucose disposal, it should be considered that insulin itself also has vascular actions under healthy conditions and this issue is a focus of investigation (10). IR, solely itself, was accused of taking part somehow in the etiology of some urologic disease such as urolithiasis and erectile dysfunction, by similar mechanism as in DM via impairing endothelial functions depending on experimental studies (9-12). The suspicion of this detrimental clinical association extends to Benign Prostate Hyperplasia/Lower Urinary Tract Symptoms (BPH/LUTS), female incontinence, male infertility and hypogonadism, and even to prostate cancer, besides the risk for some common cancer such as breast, colorectal, liver, and pancreas, if IR is evaluated together with the topic of MetS $(8,13)$.

Furthermore, in the light of current literature, there are strong evidences about that DM has negative effect on male reproductive functions in various ways as endocrine control of spermatogenesis, spermatogenesis itself or impairing penile erection and ejaculation $(1,3)$. Ali et al. reported sperm dysfunction through sperm motility defect in patients with noninsulin depended DM even in conventional spermiograms (14) (the evaluation just with conventional spermiograms may also be the limitation of the present study) but some other authors did not found any motility defect in DM without complications (15). Nevertheless, despite of the ongoing discussion about the mechanism, it is a fact that subfertility was reported in more than half of the diabetic patients (5). However, the independent negative influence of IR on human fertility was confirmed only for female infertility especially in the case of PCOS a condition that is characterized by hyperandrogenism, chronic oligo- or anovulation and polycystic ovaries. IR accompanies this clinical status with a high rate $(50-70 \%)$ and is thought to have a central pathogenic role in PCOS $(16,17)$. This relation between IR and ovarian dysfunction induced us to study the possible correlation between IR and male testicular functions through the evaluation of male sex hormones, testicular volumes and spermiograms.

IR leads compensatory hyperinsulinemia that is considered to be a promoter of the hyperandrogenism and chronic oligo- or anovulation in female with PCOS (16). In our study, gonadotropins and testosterone levels were the same as in subjects with IR or not. However, depending on the experimental and clinical studies, it is a clinical fact that DM decrease serum testosterone and increase gonadotropins (3). As opposite to our results, in a preliminary study of small groups of normal, IR and DM subjects ( $n=9,9$ and 3 respectively), Pitteloud et al. noted that increase of IR leads to a decrease in Leydig cell testosterone according to their insulin sensitivity on glucose tolerance tests (18).
In the present study there was no significant difference between mean testicular volumes (around $20 \mathrm{cc}$ for both study and control group) that can also be considered as one of the predictors of fertility. Bahk et al. concluded that the testicular volume cut off value for fertility is 18 cc (19) and testicular atrophy is a well known clinical situation in DM (20).

The proinflammatory cytokine tumor necrosis factor-a (TNF $\alpha$ ), that is a known mediator of IR, is elevated either in obese or nonobese PCOS patients. This unstable glucose metabolism leading to oxidative stress and proinflammatory status was claimed as the cause of hyperandrogenism and infertility (16). According to the present results, this explanation seemed not adaptable for subfertiles male with IR because gonadotropins and testosterone, and spermiograms were the similar in both groups, although hsCRP, as a low-grade chronic inflammatory marker, seemed significantly higher in our subjects with IR. HsCRP is found to be elevated in PCOS patients with or without metabolic syndrome (21) and, in addition, IR is a factor that elevates HsCRP (22). Thus, we think that the reason of this elevation should be attributed to the IR rather than male infertility.

Moreover, insulin, Insulin like Growth Factor 1 (IGF-1) and Growth Hormone (GH) are supposed to be part of the complex coordination function of nutrient balance and metabolic stress. Hyperinsulinemia elevates serum concentrations of free IGF-1 that can provide important trophic (anti-apoptotic) effects, leading to changes in cellular metabolism (23). Either oophorectomy or orchiectomy resulted in elevated IGF-1 levels and this suggests that IGF-1 may have some role in the infertility process (24-26). Nevertheless, further studies especially at sperm molecular level are needed to accurately confirm our preliminary results about the relation between IR and male reproductive system.

We think that our results were also independent from the effect of obesity, because BMI values of our study and control groups were similar (considered as slightly overweight for both). According to the limited number of studies, interestingly, it was supposed a loose inverse relation between male subfertility and obesity and that it occurred mostly via sperm quality and especially to the decreasing normal motility of spermatozoa (8).

Furthermore, dyslipidemia, as another component of MetS which was not present in our subjects, was accused of causing negative effect on sperm/testicular functions in both animal and human studies (27-29). However our results are far away from demonstrating the inverse relationship between MetS and male fertility with MetS components other than IR.

\section{Conclusions}

There is no doubt in the literature that DM and MetS have a "bad fame" on human fertility. In connection to this statement, IR automatically may be accused of causing detrimental effect on male fertility as a component of MetS due to hyperinsulinemic state and impaired glucose uptake as in DM2. Thus in this study, we aimed to document the possible isolate effect of IR on male reproductive system via sperm functions, serum hormone and lipid profiles 
and testicular volumes. Interestingly, according to our preliminary results, we do not find any inverse correlation between IR and male reproductive system. However, to reach a conclusion on this topic, further studies at molecular level and/or long term clinical studies of male fertility based on birth rates are needed.

\section{REFERENCES}

1. Agbaje IM, Rogers DA, McVicar CM, et al. Insulin dependent diabetes mellitus: implications for male reproductive function. Hum Reprod. 2007; 22:1871.

2. Codner E, Merino PM, Tena-Sempere M. Female reproduction and type 1 diabetes: from mechanisms to clinical findings. Hum Reprod Update. 2012; 18:568.

3. O'Neill J, Czerwiec A, Agbaje I, et al. Differences in mouse models of diabetes mellitus in studies of male reproduction. Int J Androl. 2010; 33:709.

4. Jequier AM. Is quality assurance in semen analysis still really necessary? A clinician's viewpoint. Hum Reprod. 2005; 20:2039.

5. La Vignera S, Di Mauro M, Condorelli R, et al. Diabetes worsens spermatic oxidative "stress" associated with the inflammation of male accessory sex glands. Clin Ter. 2009; 160:363.

6. Trussell JC, Legro RS. Erectile dysfunction: does insulin resistance play a part? Fertil Steril. 2007; 88:771.

7. Pauli JM, Raja-Khan N, Wu X, Legro RS. Current perspectives of insulin resistance and polycystic ovary syndrome. Diabet Med. $2011 ; 28: 1445$

8. Kasturi SS, Tannir J, Brannigan RE. The metabolic syndrome and male infertility. J Androl. 2008; 29:251.

9. Gorbachinsky I, Akpinar H, Assimos DG. Metabolic syndrome and urologic diseases. Rev Urol. 2010; 12:e157.

10. Kim JA, Montagnani M, Koh KK, Quon MJ. Reciprocal relationships between insulin resistance and endothelial dysfunction: molecular and pathophysiological mechanisms. Circulation. 2006; 113:1888.

11. Steinberg HO, Chaker $H$, Leaming $R$, et al. Obesity/insulin resistance is associated with endothelial dysfunction. Implications for the syndrome of insulin resistance. J Clin Invest. 1996; 97:2601.

12. Sánchez A, Contreras C, Martínez MP, et al. Role of neural NO synthase (nNOS) uncoupling in the dysfunctional nitrergic vasorelaxation of penile arteries from insulin-resistant obese Zucker rats. PLoS One. 2012; 7:e36027.

13. Arcidiacono B, Iiritano S, Nocera A, et al. Insulin resistance and cancer risk: an overview of the pathogenetic mechanisms. Exp Diabetes Res. 2012; 2012:789174.

14. Ali ST, Shaikh RN, Siddiqi NA, Siddiqi PQ. Semen analysis in insulindependent/non-insulin-dependent diabetic men with/without neuropathy. Arch Androl. 1993; 30:47.

15. Niven MJ, Hitman GA, Badenoch DF. A study of spermatozoal motility in type 1 diabetes mellitus. Diabet Med. 1995; 12:921.

16. González F. Inflammation in Polycystic Ovary Syndrome: underpinning of insulin resistance and ovarian dysfunction. Steroids. 2012; 77:300.

17. Pauli JM, Raja-Khan N, Wu X, Legro RS. Current perspectives of insulin resistance and polycystic ovary syndrome. Diabet Med. 2011; 28:1445.

18. Pitteloud N, Hardin M, Dwyer AA, et al. Increasing insulin resistance is associated with a decrease in Leydig cell testosterone secretion in men. J Clin Endocrinol Metab. 2005; 90:2636.

19. Bahk JY, Jung JH, Jin LM, Min SK. Cut-off value of testes volume in young adults and correlation among testes volume, body mass index, hormonal level, and seminal profiles. Urology. 2010; 75:1318.

20. Wright JR Jr, Yates AJ, Sharma HM, et al. Testicular atrophy in the spontaneously diabetic BB Wistar rat. Am J Pathol. 1982; 108:72.

21. Verit FF. High sensitive serum C-reactive protein and its relationship with other cardiovascular risk factors in normoinsulinemic polycystic ovary patients without metabolic syndrome. Arch Gynecol Obstet. 2010; 281:1009.

22. Llauradó G, Gallart L, Tirado R, et al. Insulin resistance, lowgrade inflammation and type 1 diabetes mellitus. Acta Diabetol. 2012; 49:33.

23. Clemmons DR. Metabolic actions of insulin-like growth factor-I in normal physiology and diabetes. Endocrinol Metab Clin North Am. 2012; 41:425.

24. Fogle RH, Chang L, Patel SK, et al. Increased insulin-like growth factor-1 after oophorectomy in postmenopausal women. Fertil Steril. 2008; 90:1236.

25. Sánchez-Luengo S, Fernández PJ, Romeu A. Insulin growth factors may be implicated in human sperm capacitation. Fertil Steril. 2005; 83:1064.

26. Gao F, Yang M, Luo CL, Wu XH. Local insulin-like growth factor-I of ventral prostate was upregulated during long-term castration and may function through the autocrine system. Prostate Cancer Prostatic Dis. 2011; 14:136.

27. Yamamoto Y, Shimamoto K, Sofikitis N, Miyagawa I. Effects of hypercholesterol aemia on Leydig and Sertoli cell secretory function and the overall sperm fertilizing capacity in the rabbit. Hum Reprod. 1999; 14:1516.

28. Saez Lancellotti TE, Boarelli PV, Monclus MA, et al. Hyper cholesterolemia impaired sperm functionality in rabbits. PLoS One. 2010; 18;5:e13457.

29. Ramírez-Torres MA, Carrera A, Zambrana M. High incidence of hyperestrogenemia and dyslipidemia in a group of infertile men. Ginecol Obstet Mex. 2000; 68:224.

This study has been presented (Poster no: 32) in American Society of Andrology (ASA) XXII" 2013 Testis Workshop, April 10-13, in San Antonio, Texas, USA.

\section{Correspondence}

Ayhan Verit, MD (Corresponding Author)

veritayhan@yahoo.com

Dept. of Urology - Fatih Sultan Mehmet Research and Training Hospital Icerenkoy/Atasehir - Tr- 34752 Istanbul, Turkey

Fatma Ferda Verit, MD

Associate Professor

Dept. of Obstetrics \& Gynaecology, Infertility Research \& Treatment Centre Suleymaniye Maternity, Research \& Training Hospital - Istanbul, Turkey

Halil Oncel, MD

Dept. of Urology ,Sanliurfa Research \& Training Hospital Sanliurfa, Turkey

Halil Ciftci, MD

Associate Professor

Dept. of Urology - Harran University, School of Medicine Sanliurfa, Turkey 